\title{
TOPOLOGIES ON SPACES OF VECTOR-VALUED MEROMORPHIC FUNCTIONS
}

\author{
ENRIQUE JORDÁ \\ (Received 19 December 2002; revised 30 October 2003)
}

Communicated by A. J. Pryde

\begin{abstract}
This paper presents two natural extensions of the topology of the space of scalar meromorphic functions $M(\Omega)$ described by Grosse-Erdmann in 1995 to spaces of vector-valued meromorphic functions $M(\Omega, E)$. When $E$ is locally complete and does not contain copies of $\omega$ we compare these topologies with the topology induced by the representation $M(\Omega, E) \simeq M(\Omega) \varepsilon E$ recently obtained by Bonet, Maestre and the author.
\end{abstract}

2000 Mathematics subject classification: primary 46E40; secondary 46A03, 46E05, 30D30.

\section{Introduction}

Grosse-Erdmann gives in [4] a description of the locally convex topology defined by Holdgrün in [7] on the space $M(\Omega)$ of meromorphic functions on a connected open subset $\Omega$ of $\mathbb{C}$ as a projective limit of Fréchet spaces. Grosse-Erdmann shows that the locally convex space $M(\Omega)$ is a complete Montel space and that it contains the space of holomorphic functions $H(\Omega)$ as a topological subspace. This locally convex topology seems to be natural in $M(\Omega)$. Moreover, the projective description allows him to give nice applications of the description of the dual of $M(\Omega)[4$, Section 5].

In this paper, we consider the space $M(\Omega, E)$ of meromorphic functions with values in a locally complete locally convex space $E$ endowed with the topologies which are the natural extensions of the projective and the injective limit description of the topology in $M(\Omega)$. We see that the inductive Holdgrün topology is generally finer

The research of the author was supported in part by the MCYT and the FEDER Project No. BFM 2001-2670.

(C) 2005 Australian Mathematical Society $1446-7887 / 05 \$ A 2.00+0.00$ 
than the projective topology, and that $H(\Omega, E)$ is a topological subspace of $M(\Omega, E)$ endowed with either of these two topologies. In case that $E$ is a Fréchet space the two topologies coincide.

In [2], Bonet, Maestre and the author proved that if $E$ is a locally complete locally convex space which does not contain $\omega$ as a subspace then $M(\Omega, E)$ can be canonically identified with the $\varepsilon$-product of Schwartz $M(\Omega) \varepsilon E$. We consider in $M(\Omega, E)$ the topology which makes this representation a topological isomorphism. We prove that, if $E$ has infinite algebraic dimension, this topology is strictly weaker than the projective topology. Connected with this. Let us point out that the characterization of the locally convex spaces which are locally complete [2, Proposition 2] stated in the next section permits to conclude, by a classical argument (see [8, Theorem 16.7.4]), that if $E$ is a locally complete locally convex space then $H(\Omega, E) \simeq H(\Omega) \varepsilon E$ holds topologically.

\section{Notation and preliminaries}

Throughout this paper $\Omega$ denotes a complex domain (a subset of $\mathbb{C}$ which is open and connected), and $E$ denotes a complex locally convex space. Let $I$ be an index set, the product of locally convex spaces each one of them isomorphic to $E$ is denoted by $E^{I}$, and their direct sum is denoted by $E^{(I)} . \mathbb{C}^{\mathbb{N}}$ is denoted by $\omega$ and $\mathbb{C}^{(\mathbb{N})}$ by $\varphi$.

In the sequel we will use the Pettis integral. Given a compact subset $K \subseteq \mathbb{C}$ and a function $f: K \rightarrow E, f$ is called Pettis integrable if there exists $e \in E$ such that for every $u \in E^{\prime}, u \circ f \in L^{1}(K)$ and $u(e)=\int_{K} u \circ f(z) d z$. In this case $e$ is called integral of $f$ over $K$ and we write $e:=\int_{K} f(z) d z$. It is well known that if $f$ is continuous and the closed absolutely convex hull $\overline{a c x} f(K)$ is compact, then $f$ is Pettis integrable. In this case, an easy application of the Hahn-Banach theorem shows that for every continuous seminorm $p$ on $E$ the following inequality holds

$$
p\left(\int_{K} f(z) d z\right) \leq \int_{K} p \circ f(z) d z .
$$

A function $f: \Omega \rightarrow E$ is called meromorphic if it satisfies that for each $\alpha \in \Omega$ there exists $k \in \mathbb{N}$ and there exists a sequence $\left(a_{\alpha}^{n}(f)\right)_{n=-k}^{\infty} \subset E$ such that

$$
f(z)=\sum_{n=-k}^{\infty} a_{\alpha}^{n}(f)(z-\alpha)^{n}
$$

uniformly in the compact subsets of the punctured open ball $B(\alpha, r) \backslash\{\alpha\}$ for some $r>0$. We denote by $M(\Omega, E)$ the space of $E$-valued meromorphic functions $(M(\Omega)$ if $E=\mathbb{C}$ ). For a meromorphic function $f$, we call the minimum $k \in \mathbb{N}$ satisfying (2) order of $\alpha$ at $f\left(o_{\alpha}(f)\right)$. $\alpha$ is called a pole of $f$ if $o_{\alpha}(f)>0$. The principal 
part of $f$ at $\alpha$ is $h^{\alpha}(f):=\sum_{j=1}^{o_{\alpha}(f)} a_{\alpha}^{-j}(f)(z-\alpha)^{-j}$. In case $o_{\alpha}(f)=0$, then, at $\alpha$, either $f$ is holomorphic or $f$ has a removable singularity. From the definition it follows that a meromorphic function is holomorphic except on a discrete set. The space of $E$-valued holomorphic functions on $\Omega$ is denoted by $H(\Omega, E)$. We refer to [6, Théorème 2], [1, Section 3], [11, 7.4] and [5, 4.3] for equivalent definitions of vector-valued holomorphic and meromorphic functions (see also [3, Chapter II, 2]).

In all the results presented we also assume that $E$ is locally complete. Recall that a locally convex space is called locally complete if every absolutely convex closed bounded set spans a Banach space endowed with its Minkowski gauge. In [2, Proposition 2], the locally convex spaces $E$ which are locally complete are characterized as follows: $E$ is locally complete if and only if for each $\Omega$ open in $\mathbb{R}^{n}$, for each compact subset $K$ of $\Omega$ and for each weakly $C^{1}$ function $f: \Omega \rightarrow E$, the set $\overline{\operatorname{acx}}(f(K))$ is compact in $E$. Then, for $E$ locally complete and $f \in M(\Omega, E)$, a classical argument (see [8, Theorem 16.7.2] for vector-valued holomorphic functions) shows that, for each $\alpha \in \Omega$ we can get a circle $\Gamma$ centered at $\alpha$ such that for every $k \in \mathbb{Z}$ we can write

$$
a_{\alpha}^{k}(f)=\frac{1}{2 \pi i} \int_{\Gamma}(z-\alpha)^{-k-1} f(z) d z
$$

\section{The topologies of Holdgrün and Mittag-Leffler}

In this section we define the natural extensions to $M(\Omega, E)$ of the two topologies in $M(\Omega)$ studied and shown to coincide in [4]. The proofs of some of the results are only indicated because they are simple extensions of those stated in [4] for spaces of scalar meromorphic functions. We refer to [9, Capítulo 3] for the details of these proofs.

A map $\delta: \Omega \rightarrow \mathbb{N} \cup\{0\}$ is called positive divisor on $\Omega$ if there exists a discrete subset $P_{\delta}$ of $\Omega$ such that $\delta(z)=0$ for every $z \in \Omega \backslash D$. Given a positive divisor $\delta$ on $\Omega$, we denote by $M(\Omega, \delta, E)$ the subspace of $M(\Omega, E)$ of all the functions $f$ which are holomorphic on $\Omega \backslash P_{\delta}$ and such that $o_{\alpha}(f) \leq \delta(\alpha)$ for every $\alpha \in P_{\delta}$. In these spaces we consider the topology inherited from $H\left(\Omega \backslash P_{\delta}, E\right)$ (that is, the topology of uniform convergence on the compact subsets of $\left.\Omega \backslash P_{\delta}\right)$.

PROPOSITION 3.1. For every positive divisor $\delta$ on $\Omega$, the spaces $H(\Omega, E)$ and $M(\Omega, \delta, E)$ are topologically isomorphic.

PROOF. Given a positive divisor $\delta$ on $\Omega$, we assume, without loss of generality, $\delta(\alpha) \neq 0$ if $\alpha \in P_{\delta}$. We can get a holomorphic function $g: \Omega \rightarrow \mathbb{C}$, such that for each $\alpha \in P_{\delta}, g(\alpha)=0, \lim _{z \rightarrow \alpha} g(z) /(z-\alpha)^{\delta(\alpha)} \neq 0$ and $g(z) \neq 0$ for each $z \in \Omega \backslash P_{\delta}$ 
(see [14, Theorem 15.11]). We define the linear mapping

$$
T: H(\Omega, E) \rightarrow M(\Omega, \delta, E), \quad f \mapsto \frac{f}{g} .
$$

We consider an arbitrary 0-neighbourhood in $M(\Omega, \delta, E)$ of the form

$$
U:=\left\{f \in M(\Omega, \delta, E): \sup _{z \in K} p(f(z))<1\right\},
$$

where $p$ is a continuous seminorm on $E$ and $K$ is a compact subset of $\Omega \backslash P_{\delta}$. Since $K$ is compact in $\Omega$ and $g(z) \neq 0$ for each $z \in K$, we can get $\varepsilon>0$ such that $\inf _{z \in K}|g(z)|>\varepsilon$. If we consider the 0-neighbourhood of $H(\Omega, E)$

$$
V:=\left\{f \in H(\Omega, E): \sup _{z \in K} p(f(z))<\varepsilon\right\},
$$

we have that $T(V) \subset U$, obtaining the continuity of $T$.

If we consider natural extensions to $P_{\delta}$, then the inverse mapping of $T$ is given by $T^{-1}(f)=f g$ for each $f \in M(\Omega, \delta, E)$. We consider a 0-neighborhood in $H(\Omega, E)$

$$
V:=\left\{f \in H(\Omega, E): \sup _{z \in K} p(f(z))<1\right\},
$$

where $p$ is a continuous seminorm on $E$ and $K$ is a non-empty compact subset of $\Omega$. We can assume, without loss of generality, that $K$ is non discrete and that every point in $K \cap P_{\delta}$ is an interior point of $K$. We set $K \cap P_{\delta}:=\left\{z_{i}: 1 \leq i \leq n\right\}$ (notice that the set is finite because $P_{\delta}$ is discrete in $\Omega$ and $K$ is compact), and we take $r>0$ such that the closed disc $D\left(z_{i}, r\right) \subset K$ and also $D\left(z_{i}, r\right) \cap P_{\delta}=\left\{z_{i}\right\}$ for $1 \leq i \leq n$. We consider the compact subset of $\Omega \backslash P_{\delta}$

$$
K_{1}:=K \backslash \bigcup_{i=1}^{n} B\left(z_{i}, r\right) .
$$

$K_{1}$ is non-discrete $\left(K_{1}\right.$ contains at least the union of the circles $\bigcup_{i=1}^{n} S\left(z_{i}, r\right)$ if $\left.K \cap P_{\delta} \neq \emptyset\right)$. Hence, the maximum value $M$ of the modulus of $\mathrm{g}$ in $K_{1}$ is strictly positive. We consider now the 0 -neighbourhood in $M(\Omega, \delta, E)$

$$
U:=\left\{f \in M(\Omega, \delta, E): \sup _{z \in K_{1}} p(f(z))<\frac{1}{M}\right\} .
$$

If $f$ is an $E$-valued holomorphic function defined on an open set containing the closed disc $D(a, r)$, then, for every continuous seminorm $p$ on $E$ the maximum of $p \circ f$ on $D(a, r)$ is attained at the circle $S(a, r)$. This is a consequence of the Maximum Modulus Principle for holomorphic functions and the Hahn-Banach theorem. Thus, if $f \in U, \max _{z \in K} p\left(T^{-1}(f)(z)\right)=\max _{z \in K_{1}}|g(z)| p(f(z))<1$, which is equivalent to $T^{-1}(U) \subset V$, and $T^{-1}$ is continuous. 
We denote by $\mathscr{D}_{\Omega}$ the set of all positive divisors on $\Omega$ and we consider in it the natural order $\delta_{1} \leq \delta_{2}$ if $\delta_{1}(z) \leq \delta_{2}(z)$ for every $z \in \Omega$. It is easy to check that the inclusions $i: M\left(\Omega, \delta_{1}, E\right) \rightarrow M\left(\Omega, \delta_{2}, E\right)$ are continuous for $\delta_{1} \leq \delta_{2}$. Moreover, $M(\Omega, E)=\bigcup_{\delta \in \mathscr{D}_{\Omega}} M(\Omega, \delta, E)$. Therefore we can endow $M(\Omega, E)$ with the topology given by the locally convex inductive limit $\operatorname{ind}_{\delta \in \mathscr{D}_{\Omega}} M(\Omega, \delta, E)$, which is called Holdgrün topology (see $[4,7]$ ) and it is denoted by $\tau_{\mathrm{Hol}}$. By Proposition 3.1, the space $\left(M(\Omega, E), \tau_{\mathrm{Hol}}\right)$ is an inductive limit of copies of $H(\Omega, E)$.

We denote by $H \mathscr{R}(\Omega, E)$ the space of all $E$-valued functions defined on $\Omega$ which can be written as a sum of a holomorphic function and a rational function; more precisely, $f \in H \mathscr{R}(\Omega, E)$ if there exists $g \in H(\Omega, E)$ and $\left(a_{\alpha}^{n}\right)_{\alpha \in \Omega, n \in \mathbb{N}} \in E^{(\Omega \times \mathbb{N})}$ such that

$$
f(z)=g(z)+\sum_{\alpha \in \Omega} \sum_{n \in \mathbb{N}} \frac{a_{\alpha}^{n}}{(z-\alpha)^{n}} .
$$

The functions $f \in H \mathscr{R}(\Omega, E)$ are the meromorphic functions defined on $\Omega$ and with values in $E$ which have only a finite number of poles. We consider, in the spaces $H \mathscr{R}(\Omega, E)$, the topology endowed by $H(\Omega, E) \times E^{(\Omega \times N)}$. If we denote by $\mathscr{R} \mathscr{C}_{\Omega}$ the family of all the relatively compact subdomains of $\Omega$, then $M(\Omega, E)=$ $\bigcap_{O \in \mathscr{R} \mathscr{C}_{\Omega}} H \mathscr{R}(O, E)$. Moreover, as in [4, Section 3.2], if $O_{1} \subseteq O_{2}$ are two elements of $\mathscr{R} \mathscr{C}_{\Omega}$, the linear map $T: H \mathscr{R}\left(O_{2}, E\right) \rightarrow H \mathscr{R}\left(O_{1}, E\right),\left.f \mapsto f\right|_{o_{1}}$ is easily seen to be continuous. Thus, if we consider in $\mathscr{R} \mathscr{C}_{\Omega}$ the order of the inclusion, we can endow $M(\Omega, E)$ with the topology provided by the locally convex projective limit

$$
\operatorname{proj}_{O \in \mathscr{R} \mathscr{C}_{\Omega}} H \mathscr{R}(O, E) .
$$

As Grosse-Erdmann does in the scalar case we call it the Mittag-Leffler topology and we denote it by $\tau_{M L}$. If $E$ is complete then also $H \mathscr{R}(O, E)$ is complete for each relatively compact subdomain $O$ of $\Omega$. Thus, endowed with the Mittag-Leffler topology, $M(\Omega, E)$ is complete whenever $E$ is. Moreover, $E$ is easily seen to be complemented in $\left(M(\Omega, E), \tau_{M L}\right)$. Hence the completeness of $\left(M(\Omega, E), \tau_{M L}\right)$ is equivalent to that of $E$. Again as it is done in the scalar case (see [4, Remark 2 (ii) and Theorem 1]), we can obtain an equivalent projective description: the Mittag-Leffler topology is generated by the seminorms $\|\cdot\|_{p, K, b}$, where $p$ is a continuous seminorm on $E, K \subseteq \Omega$ is compact and $b=\left(p_{\alpha}^{n}\right)_{\alpha \in K, n \in \mathbb{N}}$ is a family of continuous seminorms on $E$. Each one of these seminorms acts on a function $f \in M(\Omega, E)$ as follows (see $[4$, Theorem 1] for the scalar case):

$$
\|f\|_{K, p, b}=\sup _{z \in K} p\left(\left(f-\sum_{\alpha \in K} h^{\alpha}(f)\right)(z)\right)+\sum_{\alpha \in K, n \in \mathbb{N}} p_{\alpha}^{n}\left(a_{\alpha}^{-n}(f)\right) .
$$


REMARK. For $\left(M(\Omega, E), \tau_{M L}\right)$ to be Hausdorff one has to identify the meromorphic functions which coincide except on a discrete set. We do this throughout this paper. Hence we can always assume that $f \in M(\Omega, E)$ does not have removable singularities. This identification is implicit in [2] and [4].

A proof analogous to the one of the first part of [4, Theorem 2], but using (1), (3) and the claim, already used in the proof of Proposition 3.1, which asserts that if we consider an $E$-valued holomorphic function $f$ and a continuous seminorm $p$ on $E$ then the maximum of $p \circ f$ on a closed disc $D(\alpha, r)$ is attained on the circle $S(\alpha, r)$, permits to show the next result.

PROPOSITION 3.2. The topology $\tau_{M L}$ is coarser than $\tau_{\mathrm{Hol}}$ in $M(\Omega, E)$.

From the above proposition and the description (5) of the Mittag-Leffler topology, by using an analogous argument to the one used in [4, Theorem 4 (b)], we obtaine the next result.

PROPOSITION 3.3. $\tau_{M L}$ and $\tau_{\mathrm{Hol}}$ endow in $M(\Omega, \delta, E)$ their topology inherited from $H\left(\Omega \backslash P_{\delta}, E\right)$. In particular, $H(\Omega, E)$ is a topological subspace of $M(\Omega, E)$ endowed with any of these two topologies.

The technique which we use to show that the two topologies have the same bounded sets differs from the one used in [4] for the scalar case. We characterize the metrizable spaces admitting a continuous embedding in $\left(M(\Omega, E), \tau_{M L}\right)$, obtaining the desired result as a consequence.

PROPOSITION 3.4. Every metrizable space $F$ continuously embedded in the space $\left(M(\Omega, E), \tau_{M L}\right)$ has its image contained in $M(\Omega, \delta, E)$ for some positive divisor $\delta$ on $\Omega$.

PROOF. First we show that every subspace $H$ of $M(\Omega, E)$ either is contained in some $M(\Omega, \delta, E)$ or there exists a surjective continuous linear mapping from $H$ onto $\varphi$.

Suppose that $H$ is a subspace of $M(\Omega, E)$ such that there is no positive divisor $\delta$ on $\Omega$ for which $H \subseteq M(\Omega, \delta, E)$. We denote by $P(H)$ the subset of $\Omega$ formed by all the poles of the functions of $H$. The condition on $H$ implies that either $P(H)$ is not discrete or there exists $\alpha \in P(H)$ such that for every $n \in \mathbb{N}$ there exists a function $f \in H$ such that $o_{\alpha}(f)>n$. First we suppose that $P(H)$ is not discrete in $\Omega$. Since $\Omega$ can be written as a countable increasing union of relatively compact subdomains, we can get a relatively compact subdomain $O$ such that the cardinality of the set $(P(H) \cap O)$ is infinite. Since $O$ is relatively compact, each $f \in M(\Omega, E)$ only has finitely many poles in $O$. Thus, the choice of $O$ allows us to get inductively a 
sequence $\left(f_{k}, z_{k}, n_{k}, u_{k}\right)_{k=1}^{\infty} \subset H \times(P(H) \cap O) \times \mathbb{N} \times E^{\prime}$ such that $u_{k}\left(a_{z_{k}}^{-n_{k}}\left(f_{k}\right)\right)=1$ and $a_{z_{k}}^{-n_{k}}\left(f_{j}\right)=0$ if $k>j$ (actually we can get each $z_{k}$ as a point in which the functions $\left\{f_{j}: j<k\right\}$ are holomorphic).

Now we observe that, given a relatively compact subdomain $O$ of $\Omega$, the projective description (4) of $\left(M(\Omega, E), \tau_{M L}\right)$ yields the continuity of the projection

$$
\begin{gathered}
T_{O}:\left(M(\Omega, E), \tau_{M L}\right) \rightarrow H \mathscr{R}(O, E)=H(O, E) \times E^{(O \times \mathbb{N})}, \\
f \mapsto\left(f-\left.\sum_{\alpha \in O} h^{\alpha}(f)\right|_{O},\left(a_{\alpha}^{-n}(f)\right)_{\alpha \in O, n \in \mathbb{N}}\right)
\end{gathered}
$$

Hence, $P_{O}:\left(M(\Omega, E), \tau_{M L}\right) \rightarrow E^{(O \times \mathbb{N})}, f \mapsto\left(a_{\alpha}^{-n}(f)\right)_{\alpha \in O, n \in \mathbb{N}}$ is a surjective continuous linear mapping. The continuity of the projection $P_{O}$ implies the continuity of the mapping $p:\left(M(\Omega, E), \tau_{M L}\right) \rightarrow E^{(\mathbb{N})}$ defined by $p(f)=\left(a_{z_{k}}^{-n_{k}}(f)\right)_{k=1}^{\infty}$. Hence it is immediate that the linear mapping

$$
\begin{array}{ccc}
T: H \subseteq\left(M(\Omega, E), \tau_{M L}\right) & \rightarrow & \varphi \\
f & \mapsto & \left(u_{k}\left(a_{z_{k}}^{-n_{k}}(f)\right)_{k=1}^{\infty}\right.
\end{array}
$$

is also continuous. To see that $T$ is surjective we observe that, if we denote by $\mathbb{C}^{k}$ the subspace of $\varphi$ formed by the sequences which have vanishing all the coordinates greater than $k$, then this space is generated by $\operatorname{span}\left\{T\left(f_{j}\right): j \leq k\right\}$. A similar argument shows that if we supposed that there exists $\alpha \in P(H)$ such that there exists a sequence $\left(f_{n}\right)_{n} \subset F$ and an increasing sequence $\left(n_{k}\right)_{k} \subset \mathbb{N}$ such that $o_{\alpha}\left(f_{k}\right)=n_{k}$ then $H$ would be mapped continuously onto $\varphi$.

Now, for a metrizable locally convex space $F$, we suppose that there exists a continuous embedding $A: F \rightarrow M(\Omega)$ such that $A(F)$ is not contained in $M(\Omega, \delta, E)$ for any $\delta \in \mathscr{D}_{\Omega}$. Then, by extending to the completion, we can find a continuous and surjective linear mapping $B: \widehat{F} \rightarrow \varphi$. Thus, by the De Wilde's open mapping theorem [12, Theorem 24.30], $\widehat{F}$ is a Fréchet space with a quotient isomorphic to an infinite direct sum of copies of $\mathbb{C}$, a contradiction.

COROLLARY 3.5. The Mittag-Leffler topology and the Holdgrün topology have the same bounded sets in $M(\Omega, E)$. They are those which are contained and bounded in $M(\Omega, \delta, E)$ for some positive divisor $\delta$ on $\Omega$.

PROOF. As a consequence of Proposition 3.2, every $\tau_{\mathrm{Hol}}$-bounded set is $\tau_{M L^{-}}$ bounded. Conversely, if $B$ is a $\tau_{M L}$-bounded set, we apply Proposition 3.4 to obtain that (the linear span of the closed absolutely convex hull of) $B$ is contained in $M(\Omega, \delta, E)$ for some $\delta$ positive divisor on $\Omega$, and in this subspace both topologies coincide. 
REMARK. Proposition 3.4 together with Proposition 3.1 implies that the subspaces of $M(\Omega, E)$ endowed with any of the two topologies that we have seen in this section which are Fréchet for the induced topology are isomorphic to Fréchet subspaces of $H(\Omega, E)$.

THEOREM 3.6. If $E$ is a Fréchet space, then $\tau_{\mathrm{Hol}}=\tau_{M L}$ in $M(\Omega, E)$. In this case $M(\Omega, E)$ is a Hausdorfflocally convex space which is complete and ultrabornological.

Proof. Let $E$ be a Fréchet space. To show the coincidence between the two topologies, in view of Corollary 3.5, we only have to show that $\left(M(\Omega, E), \tau_{M L}\right)$ is bornological. This is obtained using the same method to the one used in the proof of $[4$, Lemma 2 (b)] for $M(\Omega)$. We only include a brief sketch of the proof. We fix an absolutely convex subset $U$ which absorbs $\tau_{M L}$-bounded sets and we show that there exists a relatively compact subdomain $O$ of $\Omega$, a compact subset $K$ of $O, \varepsilon>0$ and a continuous seminorm $p$ on $E$ such that if $f \in M(\Omega, E)$ does not have poles in $O$ and satisfies $\sup _{z \in K} p(f(z))<\varepsilon$, then $f \in U$. To obtain $p$ one has to use that the topology on $E$ is generated by a countable set of seminorms. Once this is proved, we observe that the subspace $\mathscr{R}(O, E)$ of $\left(M(\Omega, E), \tau_{M L}\right)$ of all the functions $f$ that can be written in the form

$$
f(z)=\sum_{\alpha \in O} \sum_{j=1}^{\infty} \frac{a_{\alpha}^{j}}{(z-\alpha)^{j}}
$$

all the vectors $a_{\alpha}^{j}=0$ but finitely many, is isomorphic to $E^{(O \times N)}$. Then it is bornological. These facts are used to show that if we denote by $V$ the set

$$
\left\{f \in M(\Omega, E): \sup _{z \in K} p\left(f(z)-\sum_{\alpha \in O} h^{\alpha}(f)(z)\right)<\varepsilon, \sum_{\alpha \in O} h^{\alpha}(f) \in U \cap \mathscr{R}(O, E)\right\}
$$

then $V$ is a 0 -neighbourhood in $\tau_{M L}$ such that $V \subseteq 2 U$.

The topological vector space $M(\Omega, E)$ is complete since it is a projective limit of complete spaces and it is bornological since it is an inductive limit of Fréchet spaces. Consequently it is ultra-bornological.

A question which arises naturally is whether the equality between Mittag-Leffler and Holdgrün topologies in $M(\Omega, E)$ holds for (DF)-spaces $E$. We have not solved this problem. However, we show below that the space $M(\Omega, \varphi)$ endowed with the Holdgrün topology is not bornological. From the projective description of the MittagLeffler topology in $M(\Omega, E)$ for a locally complete space $E$, we know that, for $n \in \mathbb{N}$, the projection over $E$ given by $P_{\alpha}^{-n}(f)=a_{\alpha}^{-n}(f)$ is continuous in $M(\Omega, E)$ endowed with its Mittag-Leffler topology, and hence also if we consider the Holdgrün topology. 
Proposition 3.7. For $\alpha_{0} \in \Omega$, the linear mapping defined on $M(\Omega, E)$ and with values in $E, f \mapsto a_{\alpha_{0}}^{0}(f)$ is continuous for the topology $\tau_{M L}$ in the space of vectorvalued meromorphic functions.

ProOF. For each relatively compact subdomain $O$ of $\Omega$, we denote by $P_{O}$ and $T_{O}$ the projections defined on $\left(M(\Omega, E), \tau_{M L}\right)$ and with values in $E^{(O \times N)}$ and $H(O, E)$ defined by $P_{O}(f)=\left(a_{\alpha}^{-n}(f)\right)_{\alpha \in O, n \in \mathbb{N}}$ and $T_{O}(f)=f-\sum_{\alpha \in O} h^{\alpha}(f)$ respectively. Both of them are continuous as a consequence of the description (4) of the MittagLeffler topology.

We fix a relatively compact subdomain $O$ of $\Omega$ containing $\alpha_{0}$ and we fix $n \in \mathbb{N}$. For each $f \in M(\Omega, E)$, we can assume without loss of generality that the function $f-h^{\alpha_{0}}(f)$ is holomorphic in a certain neighbourhood of $\alpha_{0}$ contained in $O$. This function can be developed by

$$
\left(f-h^{\alpha_{0}}(f)\right)(z)=\sum_{n=0}^{\infty} a_{\alpha_{0}}^{n}(f)\left(z-\alpha_{0}\right)^{n} .
$$

In the locally convex space $H(O, E)$, the evaluation map at a fixed point is continuous. Therefore, the continuity of $T_{O}$ implies that the mapping $T:\left(M(\Omega, E), \tau_{M L}\right) \rightarrow E$, $f \mapsto\left(f-\sum_{\alpha \in O} h^{\alpha}(f)\right)\left(\alpha_{0}\right)$ is also continuous. Moreover, by (6), we have

$$
a_{\alpha_{0}}^{0}(f)=\left(f-h^{\alpha_{0}}(f)\right)\left(\alpha_{0}\right)=T(f)+\left(\sum_{\alpha \in O, \alpha \neq \alpha_{0}} h^{\alpha}(f)\right)\left(\alpha_{0}\right) .
$$

Thus, we have only to show the continuity of the second part of the sum above. To do this we observe that this element is the composition of $P_{O}$ with the continuous linear mapping which maps every $\left(a_{\alpha}^{n}\right)_{\alpha \in O, n \in \mathbb{N}} \in E^{(O \times \mathbb{N})}$ to

$$
\sum_{\alpha \in O, \alpha \neq \alpha_{0}} \sum_{n \in \mathbb{N}} \frac{1}{\left(\alpha-\alpha_{0}\right)^{n}} a_{\alpha}^{n} \in E .
$$

REMARK. On account of the fact that in the locally convex space $H(O, E)$, the linear mapping which maps each function to its $n$-th derivative evaluated at a fixed point is continuous, a slight modification of the previous proof shows that the projections over the positive terms in the Laurent development $P_{\alpha}^{n}(f)=a_{\alpha}^{n}(f)$ are continuous on $\left(M(\Omega, E), \tau_{M L}\right)$.

To study the space $M(\Omega, \varphi)$, we first note that this space is algebraically isomorphic to $M(\Omega)^{(\mathbb{N})}$. Actually, if $f \in M(\Omega, \varphi)$ we can write $f=\left(f_{n}\right)_{n}$, and the fact that the set of zeros and poles of a non-zero meromorphic function is countable implies that $f_{n}=0$ except for finitely many $n$. We denote by $\tau_{s}$ the (bornological) topology which makes $M(\Omega, \varphi)$ isomorphic to $M(\Omega)^{(\mathbb{N})}$. 
PROPOSITION 3.8. In $M(\Omega, \varphi)$ the $\tau_{M L}$-bounded sets (and consequently the $\tau_{\mathrm{Hol}^{-}}$ bounded sets) coincide with the $\tau_{s}$-bounded sets.

PROOF. We identify $\mathbb{C}^{n}$ with the subspace of $\varphi$ formed by the sequences which have vanishing coordinates greater than $n$. If $B$ is bounded in $M(\Omega)^{(\mathbb{N})}$, then there exists $n \in \mathbb{N}$ such that $B \subset M\left(\Omega, \mathbb{C}^{n}\right)$ and we can get a positive divisor $\delta$ on $\Omega$ such that for each compact subset $K$ of $\Omega \backslash P_{\delta}$ there exists $M(K)>0$ such that for every $f=\left(f_{k}\right)_{k=1}^{n} \in B$ we have $f_{k} \in M(\Omega, \delta)$ and $\sup _{z \in K}\left|f_{k}(z)\right|<M(K)$. That is, we can choose $\delta$ such that the $n$ projections over $M(\Omega)$ which are not identically zero are contained and bounded in $M(\Omega, \delta)$. Therefore, for each $f \in B$, we have that $f \in M(\Omega, \delta, \varphi)$ and for each compact subset $K$ of $\Omega \backslash P_{\delta}$ and for each $b=\left(b_{k}\right)_{k} \in \omega$,

$$
\sup _{z \in K} \sum_{k=1}^{\infty}\left|b_{k} f_{k}(z)\right|=\sup _{z \in K} \sum_{k=1}^{n}\left|b_{k} f_{k}(z)\right|<\infty .
$$

Hence we conclude that $B$ is bounded in $M(\Omega, \delta, \varphi)$ and thereby it is $\tau_{M L}$-bounded.

Conversely, let $B$ be a $\tau_{M L}$-bounded subset of $M(\Omega, \varphi)$. We suppose that there is no $n \in \mathbb{N}$ such that $B$ is contained in $M\left(\Omega, \mathbb{C}^{n}\right)$. We choose a sequence $\left(f^{n}\right)_{n} \subset B$, with $f^{n}=\left(f_{k}^{n}\right)_{k=1}^{\infty}$ and an increasing sequence $\left(k_{n}\right)_{n} \subset \mathbb{N}$ such that $k_{n}>n$ and $f_{k_{n}}^{n} \neq 0$. The subset of $\Omega$ formed by the poles and zeros of the functions $f_{k}^{n}$ with $n, k \in \mathbb{N}$ is countable. This allows us to select $z_{0} \in \Omega$ in which every function $f_{k}^{n}$ is holomorphic and different from zero. The linear mapping

$$
\begin{aligned}
P_{z_{0}}^{0}:\left(M(\Omega, \varphi), \tau_{M L}\right) & \rightarrow \varphi \\
f & \mapsto a_{z}^{0}(f)
\end{aligned}
$$

is continuous according to Lemma 3.7. It follows that $\left(P_{z_{0}}^{0}\left(f^{n}\right)\right)=\left(f_{k}^{n}\left(z_{0}\right)\right)_{k}$ and $f_{k_{n}}^{n}\left(z_{0}\right) \neq 0$. Hence, for each $n \in \mathbb{N}$, the $k_{n}$-th coordinate of the sequence $\left(P_{z_{0}}^{0}\left(f^{n}\right)\right)$ of $\varphi$ is different from zero and therefore there is no $m \in \mathbb{N}$ for which $P_{z_{0}}^{0}(B)$ is contained $\mathbb{C}^{m}$, and then $P_{z_{0}}^{0}(B)$ is not bounded in $\varphi$, a contradiction with the continuity of $P_{z_{0}}^{0}$. Thus we can get a natural number $n$ such that $B \subset M\left(\Omega, \mathbb{C}^{n}\right)$. As, by the hypothesis and Corollary $3.5, B$ is contained and bounded in $M(\Omega, \delta, \varphi)$ for some positive divisor $\delta$ on $\Omega$, we have that the $n$ projections of $B$ over $M(\Omega)$ which are not identically zero are bounded in $M(\Omega, \delta)$, and we conclude that $B$ is $\tau_{s}$-bounded.

PROPOSITION 3.9. The space $\left(M(\Omega, \varphi), \tau_{\mathrm{Hol}}\right)$ is not bornological.

PROOF. Since $\tau_{s}$ is bornological, the above proposition implies $\tau_{\mathrm{Hol}} \leq \tau_{s}$. We have to show that $\tau_{s}$ is strictly finer. We observe that if we assume the continuity of the identity $I:\left(M(\Omega, \varphi), \tau_{\mathrm{Hol}}\right) \rightarrow\left(M(\Omega, \varphi), \tau_{s}\right)$, then the restriction of $I$ to $H(\Omega, \varphi)$ is also continuous. Hence Proposition 3.3 implies that

$$
H(\Omega, \varphi) \simeq H(\Omega)^{(\mathbb{N})}
$$


holds topologically. Since $\varphi$ is complete, $H(\Omega, \varphi)$ is isomorphic to the completion of the projective tensorial product $H(\Omega) \widehat{\otimes}_{\pi} \varphi$ (see [8, page 366]), and then (7) contradicts [13, Proposition 11.6.11].

REMARK. For a locally complete space $E$, it is not difficult to show that $M(\Omega)$ is a (complemented) subspace of $M(\Omega, E)$ endowed with either of the two topologies view. Thus, [4, Theorem $3(\mathrm{~b})]$ implies that $M(\Omega, E)$ is not metrisable nor nuclear or Schwartz. Moreover, a similar argument to the proof of [4, Theorem 3 (b)] works to show that $\left(M(\Omega, E), \tau_{M L}\right)$ is not separable. Then Proposition 3.2 implies that the space $\left(M(\Omega, E), \tau_{\mathrm{Hol}}\right)$ is not separable.

\section{The injective topology}

If $F$ is a locally convex space, as usual, we denote by $F_{c o}^{\prime}$ the topological dual of $F$ endowed with the compact open topology. For $E$ and $F$ locally convex spaces, $L_{e}\left(F_{\mathrm{co}}^{\prime}, E\right)$ denotes the space of continuous linear maps from $F_{\mathrm{co}}^{\prime}$ into $E$ endowed with the topology of the uniform convergence on the equicontinuous subsets of $F^{\prime}$. This space is called $\varepsilon$-product of Schwartz and it is denoted by $E \varepsilon F$. In [2] it is proved that if $E$ does not contain a subspace ismorphic to $\omega$, then for each $T \in L\left(E_{\mathrm{co}}^{\prime}, M(\Omega)\right.$ ) there exists $f \in M(\Omega, E)$ such that $T(u)=u \circ f$, this correspondence being an algebraic isomorphism. This representation has been used in [10] to obtain results of meromorphic extension assuming only weak meromorphic extension. In this section we study, for locally complete spaces $E$ which do not contain copies of $\omega$, the topology on $M(\Omega, E)$ which makes it isomorphic to $M(\Omega) \varepsilon E$. This topology is called the $\varepsilon$ topology and it is denoted by $\tau_{\varepsilon}$. We need some notation. Given a continuous seminorm $p$ on $E$, we denote by $U_{p, 1}$ the 0 -neighbourhood of $E$ formed by the vectors $e$ such that $p(e) \leq 1$. The polar set $U_{p, 1}^{\circ}$ is the subset of $E^{\prime}$ formed by the functionals $u$ such that $|u(e)| \leq p(e)$ for every $e \in E$. The $\tau_{\varepsilon}$-topology in $M(\Omega, E)$ is generated by the seminorms

$$
\|f\|_{p, K, b}=\sup _{u \in U_{p, 1}^{\circ}}\left(\sup _{z \in K}\left|u\left(f-\sum_{\alpha \in K} h^{\alpha}(f)\right)(z)\right|+\sum_{\alpha \in K, n \in \mathbb{N}} b_{\alpha}^{n}\left|u\left(a_{\alpha}^{-n}(f)\right)\right|\right),
$$

where $K$ is a compact subset of $\Omega, p$ is a continuous seminorm on $E$ and $b:=$ $\left(b_{\alpha}^{n}\right)_{\alpha \in K, n \in \mathbb{N}} \in \mathbb{R}_{+}^{K \times \mathbb{N}}$. Moreover, [4, Theorem 1] yields that this system of seminorms is directed; that is, given $K \subset K_{1}$ two compact subsets of $\Omega$ and $b \in \mathbb{R}_{+}^{K \times N}$ there exists $c \in \mathbb{R}_{+}^{K \times N}$ such that $\|f\|_{p, K, b} \leq\|f\|_{p, K_{1}, c}$ for each $p$ continuous seminorm on $E$ and for each $f \in M(\Omega, E)$.

First we see that the $\varepsilon$-topology is weaker than the Mittag-Leffler topology and after this we check that it is actually strictly weaker if $E$ is infinite dimensional. 
Proposition 4.1. If $E$ does not contain $\omega$ as a subspace then the topology $\tau_{\varepsilon}$ is coarser than $\tau_{M L}$ in $M(\Omega, E)$.

ProOF. To establish the claim we have to show the continuity of the algebraic isomorphism $T:\left(M(\Omega, E), \tau_{M L}\right) \rightarrow M(\Omega) \varepsilon E, f \mapsto T_{f}$, where $T_{f}(u)=u \circ f$ for each $u \in E^{\prime}$. We identify $f$ and $T_{f}$ for each $f \in M(\Omega, E)$. We fix a continuous seminorm $p$ on $E$, a compact subset $K$ of $\Omega$ and $b=\left(b_{\alpha}^{n}\right)_{\alpha \in K, n \in \mathbb{N}} \in \mathbb{B}_{+}^{K \times N}$ and we consider the continuous seminorm $\|\cdot\|_{p, K, b}$ for $\tau_{\varepsilon}$ as in (8). But the seminorm $|\cdot|$ defined on $M(\Omega, E)$ by

$$
|f|:=\sup _{z \in K} p\left(f-\sum_{z \in K} h^{\alpha}(f)\right)(z)+\sum_{\alpha \in K, n \in \mathbb{N}} b_{\alpha}^{n} p\left(a_{\alpha}^{-n}(f)\right)
$$

is continuous for the Mittag-Leffler topology according to (5). We observe that $a_{\alpha}^{-n}(u \circ f)=u\left(a_{\alpha}^{-n}(f)\right)$ holds for each $f \in M(\Omega, E)$, for each $n \in \mathbb{N}$ and for each $u \in E^{\prime}$. Hence $\|f\|_{K, p, b} \leq|f|$ for each $f \in M(\Omega, E)$.

To see that the Mittag-Leffler topology is strictly stronger that the $\varepsilon$-topology we need to use a characterization of the locally convex spaces (not necessarily locally complete) which are nuclear. For a locally convex space $E$, in $[8,15.7$ and 16.5$]$ are defined the space $l_{1}[E]$ of unconditionally $\sigma\left(E, E^{\prime}\right)$-Cauchy sequences and the space $l_{1}\{E\}$ of absolutely Cauchy sequences. By [8, Theorem 21.2.1], the locally convex spaces spaces $E$ which are nuclear are those for which $l_{1}\{E\}=l_{1}[E]$ holds algebraically and topologically. This characterization can be written as follows.

LEMMA 4.2. $E$ is nuclear if and only if for each continuous seminorm $p$ on $E$ there exists a continuous seminorm $q$ on $E$ and $\delta>0$ such that, for each finite subset $\left\{x_{1}, \ldots, x_{n}\right\}$ of $E$,

$$
\sup _{u \in U_{q, 1}^{o}} \sum_{i=1}^{n}\left|u\left(x_{i}\right)\right|<\delta \Longrightarrow \sum_{i=1}^{n} p\left(x_{i}\right)<1 .
$$

PROPOSITION 4.3. Let $E$ be a locally complete locally convex space which is not nuclear and does not contain $\omega$ as a subspace. Then the topology $\tau_{M L}$ is strictly finer than the topology $\tau_{\varepsilon}$ in $M(\Omega, E)$.

Proof. Let $E$ be a locally complete space wich does not contain $\Omega$ and such that $\tau_{\varepsilon}=\tau_{M L}$ on $M(\Omega, E)$. We fix a continuous seminorm $p$ on $E$ and an uncountable compact subset $K_{0}$ of $\Omega$. We define the following seminorm on $M(\Omega, E)$ which, according to (5), is continuous for $\tau_{M L}$

$$
\|f\|_{K_{0}, p}:=\sup _{z \in K_{0}} p\left(\left(f-\sum_{\alpha \in K_{0}} h^{\alpha}(f)\right)(z)\right)+\sum_{\alpha \in K_{0}, n \in \mathbb{N}} p\left(a_{\alpha}^{-n}(f)\right) .
$$


By the hypothesis $\|\cdot\|_{K_{0}, b}$ is also continuous for $\tau_{\varepsilon}$. Therefore we can find a seminorm $\|\cdot\|_{K_{1}, b, q}$ as in (8) for a compact subset $K_{1} \supseteq K_{0}$ of $\Omega$, a continuous seminorm $q$ on $E$ and a vector $b=\left(b_{\alpha}^{n}\right) \in \mathbb{R}_{+}^{K_{1} \times \mathbb{N}}$ such that $\|f\|_{K_{0}, p} \leq\|f\|_{K_{1}, b, q}$ for each $f \in M(\Omega, E)$. We define the sets $B_{j}=\left\{(\alpha, n) \in K_{0} \times \mathbb{N}:\left|b_{\alpha}^{n}\right|<j\right\}$. We have that $K_{0} \times \mathbb{N}$ can be written as the countable union of these sets and therefore we can get $j_{0} \in \mathbb{N}$ such that $B_{j_{0}}$ is infinite. We define $c=\left(c_{\alpha}^{n}\right) \in \mathbb{R}_{+}^{K_{1} \times \mathbb{N}}$ by

$$
c_{\alpha}^{n}= \begin{cases}j_{0} & \text { if }(\alpha, n) \in B_{j_{0}} \\ b_{\alpha}^{n} & \text { otherwise }\end{cases}
$$

If we consider the $\tau_{\varepsilon}$-continuous seminorm $\|\cdot\|_{K_{1}, c, q}$ then we have

$$
\|f\|_{K_{0}, p} \leq\|f\|_{K_{1}, b, q} \leq\|f\|_{K_{1}, c, q}
$$

for each $f \in M(\Omega, E)$. We take a sequence $\left(\alpha_{i}, n_{i}\right)_{i=1}^{\infty}$ of different points of $B_{j 0}$. For an arbitrary finite subset $\left(x_{i}\right)_{i=1}^{m}$ of $E$, we consider the meromorphic function $f(z)=\sum_{i=1}^{m}\left(z-\alpha_{i}\right)^{-n_{i}} x_{i}$, and apply (10) to $f$ to get

$$
\sup _{u \in U_{q, 1}^{o}} \sum_{i=1}^{m}\left|u\left(x_{i}\right)\right|<\frac{1}{j_{0}} \Longrightarrow \sum_{i=1}^{m} p\left(x_{i}\right)<1 .
$$

Therefore Lemma 4.2 yields that $E$ is nuclear.

Now we introduce a concept closely related to the well-known countable neighbourhood property (see [13, Definition 8.3.4]) satisfied by all the (DF)-spaces.

DEFINITION 4.4. Let $\alpha$ be a cardinal number. A locally convex space $E$ is said to satisfy the $\alpha$-neighbourhood property if for each index set $I$ with cardinality not greater than $\alpha$ and for each set $\left(p_{i}\right)_{i \in l}$ of continuous seminorms on $E$ there exists a set $\left(c_{i}\right)_{i \in I}$ of positive numbers and a continuous seminorm $p$ on $E$ such that $p_{i} \leq c_{i} p$ for every $i \in I$. Equivalently, if given a set $\left(U_{i}\right)_{i \in I}$ of 0 -neighbourhoods in $E$ there are $a(i)>0$ such that $U:=\bigcap_{i \in I} a(i) U_{i}$ is a 0 -neighbourhood in $E$.

The $\alpha$-neighbourhood property is easily checked to be inherited by subspaces. It is also clear that every normed space satisfies the $\alpha$-neighbourhood property for every cardinal number $\alpha$. We are interested in the case when $\alpha$ is the continuum cardinal $2^{\aleph_{0}}$. There exist non-normed spaces $E$ which satisfy the $2^{\kappa_{0}}$-neighbourhood property. For instance, if $I$ is an index set whose cardinality is strictly greater than $2^{\aleph_{0}}$ and we define $E$ as the space $l_{\infty}(I)$ endowed with the topology of uniform convergence on the subsets of $I$ with cardinality no greater than $2^{\aleph_{0}}$, then we have that $E$ is a non-normed space which satisfies the $2^{\aleph_{0}}$-neighbourhood property. 
LEMMA 4.5. Let $E$ be a nuclear locally convex space. Then $E$ satisfies the $2^{\aleph_{0}}$-neighbourhood property if and only if $E$ is finite dimensional.

PROOF. Every space of finite dimension is a normed space and then satisfies the $\alpha$-neighbourhood property for every cardinal number $\alpha$.

If we suppose that there exists an infinite dimensional nuclear space $E$ satisfying the $2^{\aleph_{0}}$-neighbourhood property, then we could obtain a subspace $F$ of $E$ with countable algebraic dimension. Thus $F$ is nuclear and it satisfies the $2^{\aleph_{0}}$-neighbourhood property as a subspace of $E$. The space $F$ endowed with its finest locally convex topology is isomorphic to the separable space $\varphi$. Then $F$ is also separable with its topology inherited from $E$. We select a subset $D$ of $F$ which is countable and dense. Let $\left(U_{i}\right)_{i \in I}$ be a basis of pairwise different closed 0 -neighbourhoods of $F$ such that each $U_{i}$ is the closure of its interior. We consider the map defined on $I$ and with values in $P(D)$ (the set formed by all the subsets of $D$ ) which maps each $i \in I$ to the set $U_{i} \cap D$. We observe that for each $e_{i} \in U_{i}$ and for each open set $V$ containing $e_{i}$, by the hypothesis, $V$ meets the interior of $U_{i}$. Consequently, the intersection of $V$ with the interior of $U_{i}$ is a non-empty open set and then $V \cap U_{i} \cap D \neq \emptyset$. Thus we have that $\overline{U_{i} \cap D}=U_{i}$, and from this it follows that the mapping $i \mapsto U_{i} \cap D$ is injective. Then we have that $|I| \leq 2^{\aleph_{0}}$. Now we apply that $F$ satisfies the $2^{\aleph_{0}}$-neighbourhood property to obtain that $F$ is normed. As $F$ is also nuclear, then it is finite dimensional by the Dvoretzky-Rogers theorem, a contradiction.

THEOREM 4.6. Let E be a locally complete locally convex space which does not contain $\omega$ as a subspace. The locally convex topologies $\tau_{\varepsilon}$ and $\tau_{M L}$ coincide in $M(\Omega, E)$ if and only if $E$ is finite dimensional.

Proof. By Proposition 4.3 if both topologies coincide in $M(\Omega, E)$ then $E$ is nuclear. Suppose that $E$ is nuclear. We show that the equality between the topologies is equivalent to the $2^{\aleph_{0}}$-neighbourhood property, obtaining then the desired claim by applying Lemma 4.5. Let $E$ be a space as in the hypothesis satisfying $\tau_{\varepsilon}=\tau_{M L}$ and let $\left(p_{i}\right)_{i \in I}$ be a set of continuous seminorms on $E$ such that $|I| \leq 2^{\aleph_{0}}$. Let $K$ be a compact subset of $\Omega$ with $|K|=2^{\aleph_{0}}$. We define a mapping $i: K \times \mathbb{N} \rightarrow I$, $(\alpha, n) \mapsto i(\alpha, n)$, such that $i$ is surjective. If we define $p_{\alpha}^{n}:=p_{i(\alpha, n)}$, then we have $\left\{p_{\alpha}^{n}\right\}_{n \in \mathbb{N}, \alpha \in K}=\left\{p_{i}\right\}_{i \in I}$. Let $p$ be any continuous seminorm on $E$. We define the 0 -neighbourhood in the Mittag-Leffler topology

$$
U:=\left\{f \in M(\Omega, E): \sup _{z \in K} p \circ\left(f-\sum_{\alpha \in K} h^{\alpha}\right)(f)(z)+\sum_{\alpha \in K, n \in \mathbb{N}} p_{\alpha}^{n}\left(a_{\alpha}^{-n}(f)\right)<1\right\} .
$$

By the hypothesis there exists $\delta>0$, a compact subset $K_{\mathrm{l}}$ of $\Omega$ (which we can assume to contain $K$ ), a continuous seminorm $q$ on $E$ and a set $\left(b_{\alpha}^{n}\right)_{\alpha \in K_{1}, n \in \mathbb{N}}$ of positive 
numbers such that, if we denote by $V$ the 0 -neighbourhood in $\tau_{\varepsilon}$ formed by the functions $f \in M(\Omega, E)$ which satisfy

$$
\sup _{z \in K_{1}}\left|u \circ\left(f-\sum_{\alpha \in K_{1}} h^{\alpha}(f)\right)(z)\right|+\sum_{\alpha \in K_{1}, n \in \mathbb{N}} b_{\alpha}^{n}\left|u\left(a_{\alpha}^{-n}(f)\right)\right|<\delta
$$

for each $u \in U_{q, 1}^{\circ}$, then it holds $V \subseteq U$. Looking at functions of the form $f(z)=$ $(z-\alpha)^{-n} x$ with $x \in E$ and $(\alpha, n) \in K \times \mathbb{N}$, we get that, for each $x \in E$ it holds

$$
b_{\alpha}^{n} q(x)<\delta \quad \Longrightarrow \quad p_{\alpha}^{n}(x)<1 \text {. }
$$

Hence we conclude that $E$ satisfies the $2^{\aleph_{0}}$-neighbourhood property.

Conversely, if we suppose that $E$ has the $2^{\aleph_{0}}$-neighbourhood property, by Proposition 4.1 we have only to show that $\tau_{M L} \leq \tau_{\varepsilon}$. We consider an arbitrary 0 -neighbourhood of the basis in the Mittag-Leffler topology

$$
U:=\left\{f \in M(\Omega, E): \sup _{z \in K} q\left(f-\sum_{\alpha \in K} h^{\alpha}(f)\right)(z)+\sum_{\alpha \in K, n \in \mathbb{N}} q_{\alpha}^{n}\left(a_{\alpha}^{-n}(f)\right)<1\right\},
$$

where $q$ and $q_{\alpha}^{n}$ are continuous seminorms on $E$ and $K$ is a compact subset of $\Omega$. By applying the $2^{\aleph_{0}}$-neighbourhood property to the seminorms $\left(q_{\alpha}^{n}\right)_{\alpha \in K, n \in \mathbb{N}}$ we get a continuous seminorm $p$ on $E$ and positive numbers $b_{\alpha}^{n}$ such that $q \leq p$, and $q_{\alpha}^{n} \leq b_{\alpha}^{n} p$, for each $\alpha \in K$ and each $n \in \mathbb{N}$. Thereby, if we consider the 0 -neighbourhood in $\tau_{M L}$

$$
W:=\left\{f \in M(\Omega, E): \sup _{z \in K} p\left(f-\sum_{\alpha \in K} h^{\alpha}(f)\right)(z)+\sum_{\alpha \in K, n \in \mathbb{N}} b_{\alpha}^{n} p\left(a_{\alpha}^{-n}(f)\right)<1\right\}
$$

then we have that $W \subset U$. Since $E$ is nuclear, we can apply Lemma 4.2 to obtain that there exists a continuous seminorm $r$ on $E$ such that, for each finite subset $\left\{x_{1}, \ldots, x_{n}\right\}$ of $E$, there exists $\delta>0$ such that

$$
\sup _{u \in U_{r, 1}^{o}} \sum_{i=1}^{n}\left|u\left(x_{i}\right)\right|<\delta \Longrightarrow \sum_{i=1}^{n} p\left(x_{i}\right)<\frac{1}{2} .
$$

From the fact that for every $f \in M(\Omega, E)$ and for each compact subset $K$ of $\Omega$ the subset of $K \times \mathbb{N}$ formed by the $(\alpha, n)$ for which $a_{\alpha}^{-n}(f) \neq 0$ is finite and (11), it follows that if we denote by $V$ the 0 -neighbourhood in $\tau_{\varepsilon}$ formed by the functions $f \in M(\Omega, E)$ which satisfy

$$
\sup _{u \in U_{r, 1}^{\circ}}\left(\sup _{z \in K}\left|u\left(f-\sum_{\alpha \in K} h^{\alpha}(f)\right)(z)\right|+\sum_{\alpha \in K, n \in \mathbb{N}} b_{\alpha}^{n}\left|u\left(a_{\alpha}^{-n}(f)\right)\right|\right)<\delta
$$

then $V \subset W \subset U$, concluding the proof. 
LEMMA 4.7. (a) For every positive divisor $\delta$ on $\Omega$, the topology $\tau_{\varepsilon}$ on $M(\Omega, \delta, E)$ coincides with its natural topology inherited from the space $H\left(\Omega \backslash P_{\delta}, E\right)$.

(b) The projections $P_{\alpha}^{-n}:\left(M(\Omega, E), \tau_{\varepsilon}\right) \rightarrow E, f \mapsto a_{\alpha}^{-n}(f)$, are continuous for every $\alpha \in \Omega$ and for every $n \in \mathbb{N}$.

ProOF. The description (8) of $\tau_{\varepsilon}$ implies (b) and, together with Proposition 3.2 and Proposition 4.1, also implies (a) as in [4, Theorem 4 (b)].

THEOREM 4.8. If $F$ is a Baire space continuously embedded in the space $\left(M(\Omega, E), \tau_{\varepsilon}\right)$, then there is some positive divisor $\delta$ on $\Omega$ such that the image of $F$ under the embedding is contained in $M(\Omega, \delta, E)$.

PROOF. We denote by $A$ the continuous embedding. Let $\left(O_{n}\right)_{n}$ be a sequence of relatively compact subdomains of $\Omega$ such that the closure of $O_{n}$ is contained in $O_{n+1}$ for every $n \in \mathbb{N}$ and $\Omega=\bigcup_{n} O_{n}$. We fix $n \in \mathbb{N}$ and, for $m \in \mathbb{N}$ we define $O_{n m}$ as the subset of $F$ formed by the vectors $e$ such that $A(e)$ is a meromorphic function which has in $O_{n}$ at most $m$ poles. It is clear that $F=\bigcup_{m \in \mathbb{N}} O_{n m}$. We show that $O_{n m}$ is closed in $F$. Let $e \in F \backslash O_{n m}$. There exist distinct points $\left\{\alpha_{1}, \ldots \alpha_{m+1}\right\}$ in $O_{n}$ and natural numbers $k_{1}, \ldots, k_{m+1}$ such that $P_{\alpha_{i}}^{-k_{i}}(A(e)) \neq 0$ for every $1 \leq i \leq m+1$. By Lemma 4.7 (b), the subset

$$
H:=\bigcap_{i=1}^{m+1}\left(P_{\alpha_{i}}^{-k_{i}}\right)^{-1}(\mathbb{C} \backslash\{0\})
$$

is open in $\left(M(\Omega, E), \tau_{\varepsilon}\right)$. The continuity of $A$ implies that $A^{-1}(H)$ is an open subset of $F$ containing $e$ which does not meet $O_{n m}$. Thus, since $F$ is a Baire space we conclude that there exists $m_{0} \in \mathbb{N}$ such that $O_{n, m_{0}}$ has non-empty interior. We observe that it holds $O_{n m}+O_{n m} \subset O_{n 2 m}$ and $\lambda O_{n m}=O_{n m}$ for each $\lambda \in \mathbb{C} \backslash\{0\}$ to conclude that there exists a 0 -neighbourhood in $O_{n, 2 m_{0}}$. This yields that $F=O_{n, 2 m_{0}}$. It is not difficult to show that, for each subspace $G$ of $M(\Omega, E)$, if $f \in G, \alpha \in O_{n}$ is not a pole of $f$ and there exists $g \in G$ such that $\alpha$ is a pole of $g$, then there exists $\varepsilon>0$ such that if $\beta \in \mathbb{C}$ satisfies $0<|\beta|<\varepsilon$ then each pole of $f$ in $O_{n}$ and also $\alpha$ are poles of $f+\beta g \in G$. Thus, the equality $F=O_{n, 2 m_{0}}$ yields that the subset of $O_{n}$ formed by the points which are poles of the meromorphic functions which belong to $A(F)$ is finite. Hence, if we denote by $P(F)$ the subset of $\Omega$ formed by the points which are poles of meromorphic functions which can be written as $A(e)$ with $e \in F$, we have that $P(F)$ is discrete in $\Omega$.

Now we fix $\alpha \in P(F)$. We define

$$
F_{\alpha}^{n}:=\bigcap_{k=n}^{\infty}\left(P_{\alpha}^{-k} \circ A\right)^{-1}(0) .
$$


Each $F_{\alpha}^{n}$ is a closed vector subspace of $F$. Moreover, we have $F=\bigcup_{n=1}^{\infty} F_{\alpha}^{n}$. Since $F$ is a Baire space, we can get $n(\alpha) \in \mathbb{N}$ such that $F=F_{\alpha}^{n(\alpha)}$. Then we can define $\delta: \Omega \rightarrow \mathbb{N} \cup\{0\}$ by $\delta(\alpha)=n(\alpha)$ if $\alpha \in P(F)$ and $\delta(\alpha)=0$ otherwise. We have that $\delta$ is a positive divisor on $\Omega$ and $F \subset M(\Omega, \delta, E)$.

COROLLARY 4.9. If $E$ is a locally complete locally convex space which does not contain a subspace isomorphic to $\omega$, then the subsets of $M(\Omega, E)$ which are $\tau_{\varepsilon^{-}}$ bounded are the same ones that the $\tau_{M L}$-bounded (or $\tau_{\mathrm{Hol}}$-bounded) sets.

ProOF. $\left(M(\Omega, E), \tau_{\varepsilon}\right)$ is the locally complete space $M(\Omega) \varepsilon E$ (since $M(\Omega)$ and $E$ are locally complete). Therefore the conclusion follows as in Corollary 3.5 by applying Lemma 4.7 (a) and Theorem 4.8.

COROLLARY 4.10. If $E$ is an infinite dimensional locally complete locally convex space which does not contain copies of $\omega$ then $\left(M(\Omega, E), \tau_{\varepsilon}\right)$ is not bornological.

\section{Acknowledgements}

This work is part of the author's PhD Thesis, written under the supervision of J. Bonet and M. Maestre. The author wishes to thanks them. Without their guidance and advice, this work would have been impossible.

\section{References}

[1] J. Bochnack and J. Siciak, 'Analytic functions in topological vector spaces', Studia Math. 39 (1971), 77-112.

[2] J. Bonet, E. Jordá and M. Maestre, 'Vector-valued meromorphic functions', Arch. Math. 79 (2002), 353-359.

[3] K.-G. Grosse-Erdmann, The Borel Okada theorem revisted (Habilitation, Fernuniv. Hagen, 1993).

[4] - 'The locally convex topology on the space of meromorphic functions', J. Austral. Math. Soc. (Series A) 59 (1995), 287-303.

[5] - 'A weak criterion for vector-valued holomorphy', Math. Proc. Cambridge Phil. Soc. 136 (2004), 399-411.

[6] A. Grothendieck, 'Sur certains espaces de fonctions holomorphes', J. Reine Angew. Math. 192 (1953), 35-64.

[7] H. S. Holdgrün, 'Fastautomorphe Funktionen auf komplexen Räumen', Math. Ann. 203 (1973), $35-64$.

[8] H. Jarchow, Locally convex spaces (B. G. Teubner, Stuttgart, 1981).

[9] E. Jordá, Espacios de funciones meromorfas (Ph.D. Thesis, Universidad Politécnica de Valencia, Spain, 2001).

[10] _ 'Extension of vector-valued holomorphic and meromorphic functions', Bull. Belgian Math. Soc. 12 (2005), 1-17. 
[11] A. Kriegl and P. W. Michor, The convenient setting of global analysis (American Mathematical Society, Providence, RI, 1997).

[12] R. Meise and D. Vogt, Introduction to functional analysis (Clarendon, Oxford, 1997).

[13] P. Pérez Carreras and J. Bonet, Barrelled locally convex spaces (North-Holland, Amsterdam, 1987).

[14] W. Rudin, Real and complex analysis, 3rd edition (McGraw-Hill, New York, 1991).

Departamento de Matemática Aplicada

E. Politécnica Superior de Alcoy

Universidad Politécnica de Valencia

Plaza Ferrándiz y Carbonell 2

E-03801 Alcoy (Alicante)

Spain

e-mail: ejorda@mat.upv.es 